\title{
Numerical Study of Laminar Flame Speed of Fuel-Stratified Hydrogen/Air Flames
}

\author{
Xian Shi ${ }^{\mathrm{a}, *}$, Jyh-Yuan Chen ${ }^{\mathrm{a}}$, Zheng Chen ${ }^{\mathrm{b}}$ \\ ${ }^{a}$ Department of Mechanical Engineering, University of California - Berkeley, Berkeley, CA 94720, USA \\ ${ }^{b}$ Department of Mechanics and Engineering Science, College of Engineering, Peking University, Beijing 100871, China
}

\begin{abstract}
Numerical studies on hydrogen/air stratified flames in 1-D planar coordinate are performed using a time-accurate and space-adaptive numerical solver A-SURF. A step change in equivalence ratio is initialized as fuel stratification. Flame characterizations including fuel consumption speed and flame front propagation speed are compared between stratified flames and corresponding homogeneous flames. Two transport models, with equal diffusivity and mixture-average diffusivity assumptions respectively, are considered. With equal diffusivity assumption and stratification thickness larger than flame thickness, local fuel consumption speeds of stratified and homogeneous flames are identical, indicating that neither thermal effect nor chemical effect is present in stratified flames. When stratification thickness is reduced to the order of flame thickness, the difference between local fuel consumption speeds of stratified and homogeneous flames is caused by chemical effect due to different level of $\mathrm{H}$ radical in burnt gas. The same mechanism also leads to the difference between local fuel consumption speeds with mixture-average diffusivity assumption. In addition, preferential diffusion of $\mathrm{H}$ radical further increases the difference. The difference between flame front propagation speeds of stratified and homogeneous flames is mainly caused by additional heat release in the burnt gas with equal diffusivity assumption, while the difference with mixture-average diffusivity assumption is mainly caused by local chemical effect. Hydrodynamic effect due to fluid continuity on flame front propagation speeds is observed in both transport models. Additionally, with increasing stratification thickness, both local chemical and hydrodynamic effect are reduced. No significant lean flammability extension of hydrogen/air mixture is introduced by fuel stratification.
\end{abstract}

Keywords: Hydrogen/air stratified flame, Laminar flame speed, Fuel consumption speed, Flame front propagation speed, Differential diffusion

\section{Introduction}

Fuel-stratified, overall-lean combustion has become one of the potential internal combustion engine techniques $[1,2]$. While lean combustion leads to low $\mathrm{NO}_{\mathrm{x}}$ emission without losing combustion efficiency [3, 4], charge stratification improves ignitability and flame stability [5, 6]. In addition, fuel stratification is also regarded as a useful technique to control combustion phasing and expand load limits in piston engines[7]. Despite its wide application in industries, fundamental understanding of stratified flames is still incomplete, which impedes the development of advanced direct fuel injection control strategies. In particular, as laminar flame speed of stratified charge is a key factor

\footnotetext{
${ }^{*}$ Corresponding author.

Email address: xshi@berkeley.edu (Xian Shi)
}

to determine the interaction between fuel auto-ignition events and flame propagation in stratified compression ignition engines, how laminar flame speed of stratified flame varies compared to the corresponding homogeneous flames is crucial but still not well understood.

There are two typical types of stratified flames: stratified flames propagating perpendicular to the mixture stratification layer, or along the layer. The first type of stratified flames is often investigated with a detailed discussion on structure of tribrachial triple flames $[8,9,10]$, as a diffusion flame branch is created along the stoichiometric mixture fraction line. In comparison, the second type of stratified flames, when stratified flames propagate along the mixture stratification layer, only has the premixed flame branch. It is relatively complicated as flame characteristics are transient and alter in time due to flame passing through different local mixture compositions across the stratification layer. This 
study focuses on the latter stratified flames, which propagate along fuel stratification layer.

There have been extensive theoretical, experimental and numerical research on stratified flames propagating along mixture stratification layer: Effects of stratification on flammability limit [11, 12, 13], flame propagation speed $[14,15]$, flame structure $[16,17,18]$ and etc. have been investigated, with regard to different fuel components including hydrogen [13], methane $[14,15]$ and iso-octane [16]. As to the effect of stratification on laminar flame speeds, two mechanisms have been proposed to explain the behavior of stratified flames. A. Pires Da Cruz et al. [19] conducted 1-D planar, unsteady laminar flame simulations on stratified methane/air mixture with detailed chemistry. Both rich and lean mixtures undergoing through positive or negative equivalence ratio gradients with regard to flame propagation direction were investigated. They suggested that the propagation of lean stratified flames is influenced mainly by burned gas temperature, i.e., thermal effect. When flame is burning from stoichiometry to lean, additional heat and composition fluxes from hotter products accelerate combustion at the flame front compared to the corresponding homogeneous flames at the same equivalence ratio. For rich stratified flames, the propagation is additionally influenced by production and consumption of molecular hydrogen in the flame front and the burnt gas, i.e., chemical effect. This causes the stoichiometric to rich flames to slow down and the rich to stoichiometric flames to accelerate, compared to homogeneous flames. However, only the fuel consumption over the entire domain was investigated and the impact of stratification on local flame characteristics was missing. In the content of computational fluid dynamics (CFD), local fuel consumption is especially more relevant in internal combustion engine applications and turbulent combustion. Moreover, although the differences in temperature and species distribution between stratified flames and homogeneous flames were reported, how they actually led to the difference in flame characteristics is not fully understood. In the second mechanism study, by performing numerical simulation of methane/air counterflow stagnation flames, Zhou and Hochgreb [20] confirmed that methane/air stratified flames are primarily dominated by the diffusion of heat under lean conditions, and diffusion of $\mathrm{H}_{2}$ under rich conditions. However, these observations are only valid in the cases of certain strain rates under steady state. How stratification relates to unstretched propagating flames is still unclear. In fact, stratified flames are difficult to characterize, e.g. location of flame front, equivalence ratio at flame front and definition of flame speeds. Especially when differential diffusion exists, gas composition at the flame front can be quite different from that of unbrnt mixture, making the characterization even harder. Furthermore, observation from one particular fuel stratification might also not hold for another fuel, as different fuel species burn through different chemical pathways. As one of the fundamental combustible mixtures, hydrogen/air flame generates intermediate radicals, which appear in almost all hydrocarbon flames. Therefore a through understanding of hydrogen/air flames is essentially important to establish the first picture of fuel stratification concept.

The objective of this study is to understand how hydrogen/air stratified flame behaves differently in comparison to homogeneous flames. More specifically, the following questions are to be answered:

1. What is the detailed mechanism that stratification introduces thermal effect or chemical effect, if any, leading to variation of flame characteristics between stratified flames and homogeneous flames?

2. What is the role of differential diffusion of chemical species in stratified flames? Is it related to the chemical effect?

3. How do flame characteristics respond to different degrees of stratification?

To answer these questions, fuel consumption speed as well as flame front propagation speed are used to quantify the differences between stratified and homogeneous flames. Two transport models with equal diffusivity and mixture-average diffusivity assumptions respectively are considered and analyzed. The effect of stratification thickness on stratified flames as well as the potential on lean flammability extension are also investigated.

\section{Numerical Model and Setup}

The present numerical model represents hydrogen/air flame propagation in one-dimensional (1-D) planar coordinate with one end closed and the other open, as sketched in Figure 1. The flame is initialized at the closed end and propagates toward the open end. Stratification is introduced by specifying a step change in the 
initial equivalence ratio profile. The simulated condition is analogous to constant-volume bomb experiments where ignition occurs in the center and flame propagates outwardly [21], or to tube experiments where flame propagates in a pipe from its closed bottom to the open end [22].

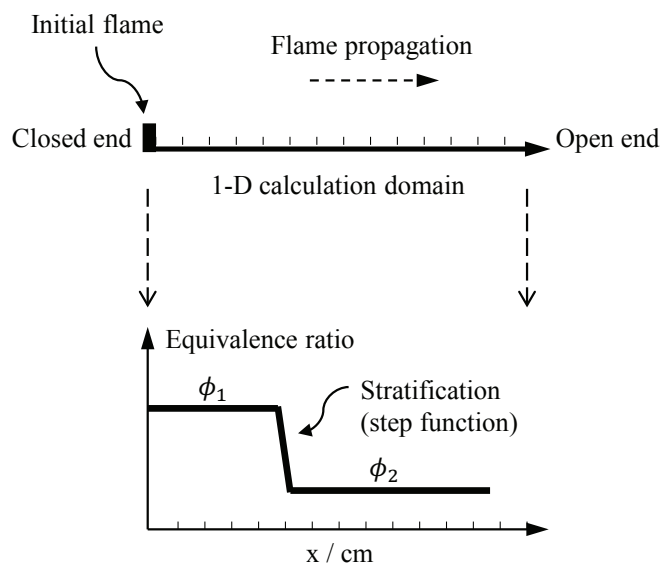

Figure 1: Schematic of stratified flame propagating in 1-D planar coordinate.

The numerical simulation is carried out by a timeaccurate and space-adaptive numerical solver named the Adaptive Simulation of Unsteady Reacting Flow - A-SURF, originally developed by the co-author of this paper [23]. The unsteady compressible NavierStokes equations along with energy and species conversation equations for a multi-species reactive mixture in 1-D planar coordinate are solved. A 9-species detailed chemistry mechanism of hydrogen/air mixture [24] is used and the chemical kinetics are solved by CHEMKIN packages [25]. Dynamically and locally adaptive mesh refinement(AMR) based on local temperature gradients is also applied. A-SURF has been successfully applied to many previous studies of homogeneous flames [26, 27, 28, 29] demonstrating highfidelity simulation capability. For this study of stratified flames, three major modifications have been made: 1) application of transport model with equal diffusivity assumption, 2) specification of the initial equivalence ratio profile through a step change and 3) additional AMR based on local equivalence ratio gradient. All the cases reported here were conducted in a 50 planar $\mathrm{cm}$ domain. The initial temperature is $300 \mathrm{~K}$ while pressure is $1 \mathrm{~atm}$. Reflective boundary condition is applied at the first grid point to simulate the closed end, while transmissive boundary condition at the last grid point to simulate open end. The time step is specified by a fixed CFL number of 0.25 . Due to both temperature and equivalence ratio AMR, both sizes of grid cells and time step are dynamically adjusted during the run.

The flame is initialized by imposing burnt gas composition and temperature profiles from CHEMKIN PREMIX [30] to the grid points on the left side so that the flame can be established with relatively small time duration and the initial unsteadiness is minimized. The step change in equivalence ratio is placed a certain distance away from the initial flame so that the flame will achieve a steady state when it reaches the stratification. The step itself is specified by a sharp change between $\phi_{1}$ and $\phi_{2}$ across several grid points, as shown in Figure 1. Details on stratification thickness will be discussed in the following sections.

\section{Flame Characterizations in Stratified Flames}

Flame characterizations in stratified mixtures are not as well-defined as those in homogeneous mixtures due to the composition variation across the flame zone [19]. In order to make meaningful comparison between stratified and homogeneous flames, several flame characterizations are specifically defined and explained in the following.

\subsection{Equivalence Ratio at Flame Front, $\phi_{f}$}

Flame front location is a fundamentally significant feature for describing non-stationary flames. In experimental studies, flame front may be defined by the maximum density gradient measured using Schlieren technique [31], or a threshold level of representative species/radical concentration measured by laser induced fluorescence technique [32]. Following [20], flame front in this numerical study is defined as the location at which heat release rate is maximum.

Next the equivalence ratio at flame front should be reasonably defined to achieve fair comparison between stratified and homogeneous flames. For stratified flames, it is inappropriate to define the equivalence ratio based on upstream unburnt gas mixture. Therefore the equivalence ratio at flame front used here is based on local element composition instead of actual fuel/air ratio, similar to [20]:

$\phi_{f}=\frac{X_{H}}{2 X_{O}}$,

where $X_{H}, X_{O}$ denote the element mole fraction of $\mathrm{H}$ and $\mathrm{O}$ at the flame front respectively. For homogeneous flames, the equivalence ratio based on unburnt gas mixture $\phi_{u}$ will be equal to $\phi_{f}$ when equal diffusivity is 
assumed. In contrast, with mixture-average diffusivity assumption, $\phi_{f}$ may deviate from $\phi_{u}$, even for homogeneous flames, due to differential diffusion of species. Therefore in this study, stratified flames will be compared to their corresponding homogeneous flames with the same equivalence ratio at flame front $\phi_{f}$.

\subsection{Stratification thickness, $\delta_{S}$}

Since the step change in equivalence ratio is placed at a certain distance from the closed end, during the time period when flame propagates from the initial flame position to the step, the stratification layer has grown due to mass diffusion. In order to quantify the degree of stratification when the flame front reaches the stratified region, stratification thickness $\delta_{S}$, or referred as mixing layer thickness, is defined as shown in Figure 2 and given as following:

$\delta_{S}=\frac{\left|\phi_{1}-\phi_{2}\right|}{\left|\frac{d \phi}{d x}\right|_{\max }}$.

Different stratification thicknesses can be achieved by placing the initial step change at different locations relative to the closed end. Note that this study mainly focuses on cases where stratification thickness is at the same order magnitude or larger compared to flame thickness, which is approximately $0.04 \mathrm{~cm}$ based on peak temperature gradient [33]. With this being said, stratified flames propagate through a natural equivalence ratio gradient where burnt gas temperature and composition are different from those of corresponding HF. Stratification thickness in all cases demonstrated in this study is of the order of $0.1 \mathrm{~cm}$ if not specified otherwise.

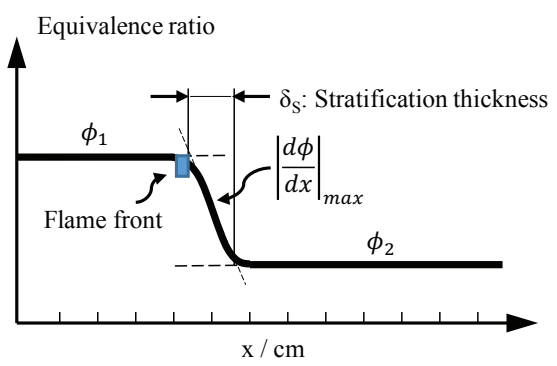

Figure 2: Schematic of stratification thickness.

\subsection{Fuel consumption speed, $S_{c}$}

Based on numerical simulation results, laminar flame speeds can be determined through the profile of flame properties across the flame, such as temperature variation and species distribution [34]. From the fuel consumption point of view, laminar flame speed can be represented by the fuel consumption speed as defined by $[29,35]$ :

$$
S_{c}=-\frac{1}{\rho_{u} Y_{F}^{u}} \int \dot{\omega}_{F} d x
$$

where $Y_{F}^{u}$ is the mass fraction of fuel species in the unburnt gas; $\rho_{u}$ is the unburnt gas density and $\dot{\omega}_{F}$ is the fuel consumption rate. Theoretically, $Y_{F}^{u}-Y_{F}^{b}$ may be used in Equation 3 instead of $Y_{F}^{u}$, especially for rich mixture flames [36]. However in stratified flames, $Y_{F}^{b}$ is not well-defined as the burnt gas is stratified. Even for homogeneous flames, uncertainty exists in $Y_{F}^{b}$ as the burnt gas composition of propagating flames can deviate from the corresponding equilibrium composition [37]. Therefore, $Y_{F}^{u}$ is used for both stratified and homogeneous flames.

As fuel species are not only consumed around the flame front but also continuously reacting in the burnt gas due to finite rates of chemistry, the integral limits used in Equation 3 will determine the scope of fuel consumption speed. For homogeneous flames, the limits can range from negative to positive infinity. However for stratified flames, the integral would include fuel consumption from burnt gas with different equivalence ratios, causing ambiguity when compared to the corresponding homogeneous flames with the same $\phi_{f}$. Therefore, different integral limits are applied to distinguish between local and global fuel consumption speeds. In Figure 3, both 0\% and 5\% cutoff integral limits are illustrated. While $0 \%$ cutoff represents the integral over the entire domain, 5\% means that the integral will be conducted only over the domain where local heat release rate exceeds $5 \%$ of the maximum heat release rate. In this way, 5\% cutoff fuel consumption speed is considered as the local fuel consumption speed and therefore represents local flame characteristics. 


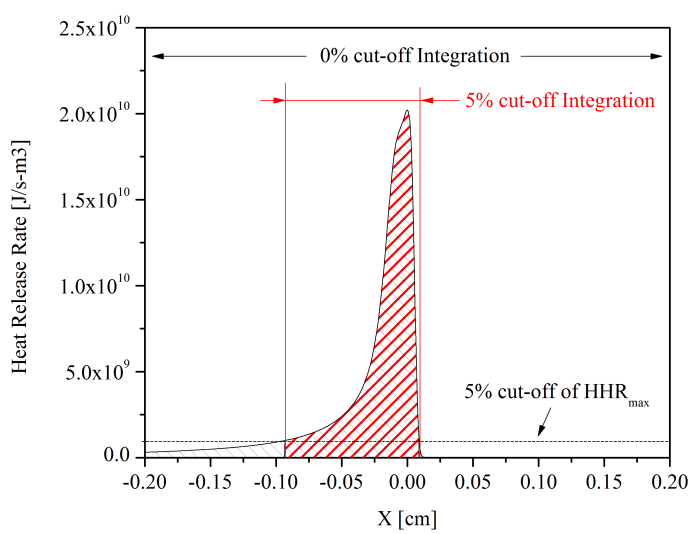

Figure 3: Fuel consumption speed integration with different cutoff integral limits with regard to maximum heat release rate.

\subsection{Flame front propagation speed, $S_{b}$}

In experimental studies, laminar flames can be directly characterized by a global behavior of the flame, such as flame front movement speed in outwardly propagating flames or equivalent fluid velocity in opposed jet flames. In this study, flame front propagation speed, $S_{b}$, is defined as the physical moving speed of the flame front in the calculation domain. Note that $S_{b}$ can be directly measured from experiments and is related to $S_{L}$, laminar flame speed relative to unburnt mixture. In homogeneous flames, the relation between $S_{b}$ and $S_{L}$ can be expressed as

$S_{b}=\frac{\rho_{u}}{\rho_{b}} S_{L}$,

where $\rho_{u}$ and $\rho_{b}$ represent density of unburnt and burnt mixture. Similar to $Y_{F}^{u}, \rho_{b}$ is not well-defined in stratified flames as well as propagating homogeneous flames. Therefore $S_{L}$ cannot be unambiguously derived from the above relation.

\subsection{Fluid expansion speed, $S_{\text {exp }}$}

Propagation of flame front is resulted from not only the burning velocity that flame propagates into unburnt mixture, but also the expansion of burnt gas due to additional heat release. It is useful to define burnt gas expansion speed for assessing the fluid expansion effect on the flame front propagation speed. In order to formulate this speed definition, each computational cell is regarded as a single 1-D control mass, which can only expand or shrink but not exchange mass with neighbor cells. The cell size changing rate represents the gas expansion due to heat release. Therefore, the sum of the size changing rates of all cells, from closed end to flame front, will represent the total burnt gas expansion. If the burnt gas heat release and consequential expansion differ between SF and HF, the corresponding differences will be observed in fluid expansion speeds as well as flame front propagation speeds. To calculate the cell size changing rate, mass conservation is applied to each single 1-D cell:

$d\left(\rho x_{c}\right)=0$,

where $x_{c}$ stands for the cell size. Taking the derivative of the above equation with respect to time yields:

$\frac{d x_{c}}{d t}=-\frac{x_{c}}{\rho} \frac{d \rho}{d t}$.

Based on ideal gas law with constant pressure, density changing rate can be written as temperature changing rate. Molecular weight of the mixture is assumed unchanged as burnt gas composition has approximately reached equilibrium. As temperature increase is related to heat release, temperature changing rate can be further expressed as a function of local heat release rate, density and specific heat. Therefore, the size expansion rate can be eventually written as

$\frac{d x_{c}}{d t}=\frac{x_{c}}{T} \frac{d T}{d t}=\frac{x_{c}}{\rho c_{p} T} \frac{d q}{d t}=\frac{x_{c} \dot{q}}{\rho c_{p} T}$,

The fluid expansion speed is then determined by integrating the expansion rate of all grid cells up to the location of flame front.

$S_{\text {exp }}=\int_{0}^{x_{f}} \frac{\dot{q}}{\rho c_{p} T} d x$,

where $x_{f}$ stands for the location of flame front. Numerically it can be also expressed as

$S_{\text {exp }}=\sum^{f} \frac{x_{c} \dot{q}}{\rho c_{p} T}$.

\subsection{Diffusion Speed, $V$}

In order to quantify the thermal and chemical effects introduced by the burnt gas of stratified flames, thermal and species diffusion speeds are defined as following:

$$
\begin{aligned}
V_{T} & =-D_{T} \frac{d \ln T}{d x}, \quad D_{T}=\frac{\lambda}{\rho c_{p}} \\
V_{k} & =-D_{k} \frac{d \ln X_{k}}{d x},
\end{aligned}
$$


where $D_{T}$ is thermal diffusivity and $D_{k}$ is mass diffusivity of species $k$. These quantities are used in the energy and species conservation equations and represent how fast heat and chemical species diffuse relative to fluid velocity. Absolute diffusion speed is thereby defined as diffusion speed relative to the lab frame, i.e., diffusion speed plus local fluid velocity. Thermal or chemical effects on local flame characteristics will exist only if heat or mass diffusion on the basis of fluid velocity are fast enough to catch up the propagation of flame front.

\section{Results and Discussion}

In piston engines, fuel stratification is created by the injection of fuel into the combustion chamber. Ignition is often initiated at a rich mixture, creating stratified flames propagating from rich to lean. Therefore, rich-to-lean stratified flame is the most common stratified flame in practical applications and thus the primary focus in understanding the mechanism of stratified flames. Other stratification cases are also considered in this study as theoretical references.

\subsection{Equal Diffusivity Results (Le =1)}

In this section, equal diffusivity is assumed: mass difussivity of all chemical species are set to the thermal difussivity, i.e. Lewis number is set to one $(L e=1)$. Thermal diffusivity is calculated on the basis of local mixture properties. This assumption is a useful tool to separate thermal and chemical effects when studying stratified flames, as it suppresses differential diffusions and examines whether only thermal effect will lead to any difference between SF and HF. As differential diffusion is excluded, $\phi_{f}$ is equal to $\phi_{u}$ in homogeneous flames.

Figure 4 compares fuel consumption speed results calculated with both $0 \%$ and $5 \%$ cutoffs, of both stratified flames (SF) and homogeneous flames (HF). The stratified flame is propagating from a homogeneous charge of equivalence ratio 2.0 (rich) to that of 0.5 (lean) through the step change. Note that the fuel consumption speeds based on $0 \%$ cutoff peak at $\phi_{f}=1.0$, and some certain differences are observed between SF and HF. In contrast, the $5 \%$ cutoff results peak at $\phi_{f}=1.3$ and collapse on top of each other, indicating that there is no difference in local fuel consumption between SF and HF. To interpret these results, detailed flame characteristics near flame front such as temperature and species distributions are investigated. Figure 5 shows the heat release rate, temperature, species mole fraction and their corresponding diffusion speeds profiles of SF and $\mathrm{HF}$ when $\phi_{f}=1.2$. The position at $x=0$ represents the location of flame front. Thus the region of $x<0$ represents burnt gas near the flame and the other side is unburnt. In Figure 5a, heat release rate profiles are identical between SF and HF, which agree with the $5 \% S_{c}$ results. Temperature in the burnt gas of SF seems to be slightly lower than that of HF as SF propagates from richer mixture with lower adiabatic flame temperature. In Figure 5b, more $\mathrm{H}$ and $\mathrm{H}_{2}$ but less $\mathrm{OH}$ are found in the burnt gas of SF than HF, while they are almost identical near flame front. These temperature and species differences agree with previous results reported in [19], but certainly do not lead to changes in local flame characteristics as heat release and $5 \% S_{c}$ results are identical between SF and HF. If diffusion of heat and species in the burnt gas are not fast enough to catch up with the propagation of flame front, there will be no effect introduced by these differences. Figure $5 c$ shows the $a b-$ solute thermal and species diffusion speeds compared to $S_{b}$, the flame front propagation speed. The absolute thermal diffusion speed, labeled as $T$, is found much slower than $S_{b}$, indicating heat diffusion is much slower than flame front propagation. Therefore, extra heat diffusion due to the difference in burnt gas temperature between SF and HF is not able to influence local fuel consumption. In comparison, absolute species diffusion speeds are also overall slower than $S_{b}$ except that $\mathrm{H}$ and $\mathrm{OH}$ diffusion is faster than $S_{b}$ in the region near flame front. However, in the same region near flame front, $\mathrm{H}$ and $\mathrm{OH}$ mole fraction profiles are identical between $\mathrm{SF}$ and $\mathrm{HF}$ as shown in Figure 5b. Therefore, the difference in species distribution is also not able to influence local fuel consumption. In short, with equal diffusivity assumption, the above analysis reveals that neither thermal effect nor chemical effect impacts local fuel consumption.

The difference observed in the $0 \%$ cutoff results is due to additional fuel consumption in the burnt gas dominated by burnt gas temperature, since chemical reactions do not completely finish and continue to happen after the flame has passed through. As SF propagates firstly from rich to stoichiometry, the burnt gas of SF has lower adiabatic temperature than that of HF. Therefore, less fuel per unit mixture is consumed, which makes less contribution to the overall $0 \%$ fuel consumption speed of SF. When SF continues to propagate from stoichiometry to lean, higher burnt gas temperature is achieved. As a result, the gap between SF and HF results starts to decrease and eventually SF catches up with HF. This explains the trend that $0 \%$ cutoff $S_{c}$ of SF is firstly slower than that of HF but later able to catch up with HF. 


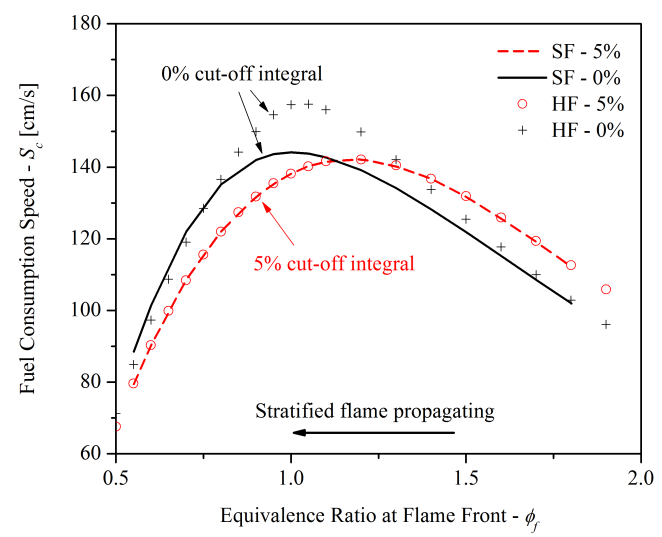

Figure 4: Fuel consumption speeds with $0 \%$ cutoff and $5 \%$ cutoff of $\mathrm{SF}$ and HF, when SF propagates from $\phi_{f}=2.0$ to $\phi_{f}=0.5, \delta_{S}=0.1$ $\mathrm{cm}$, with equal diffusivity assumption.

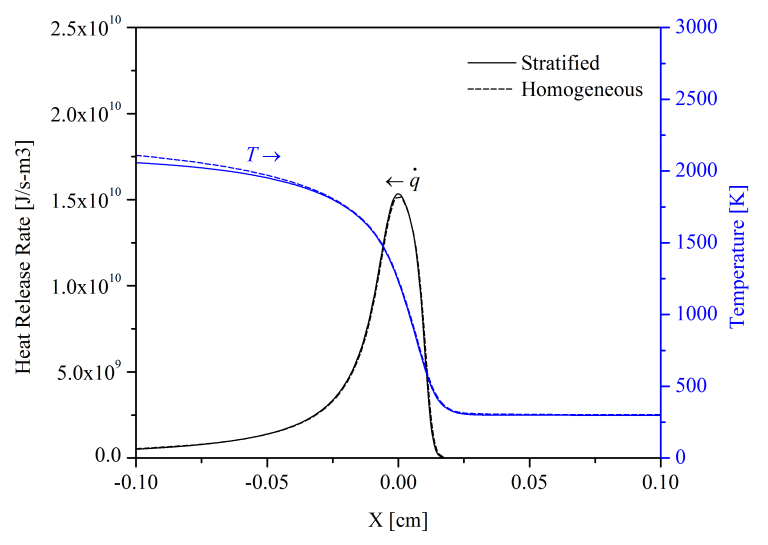

(a)

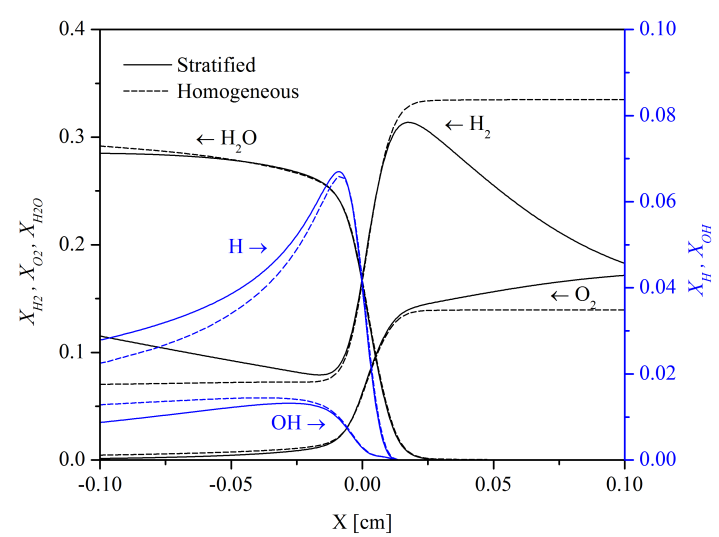

(b)

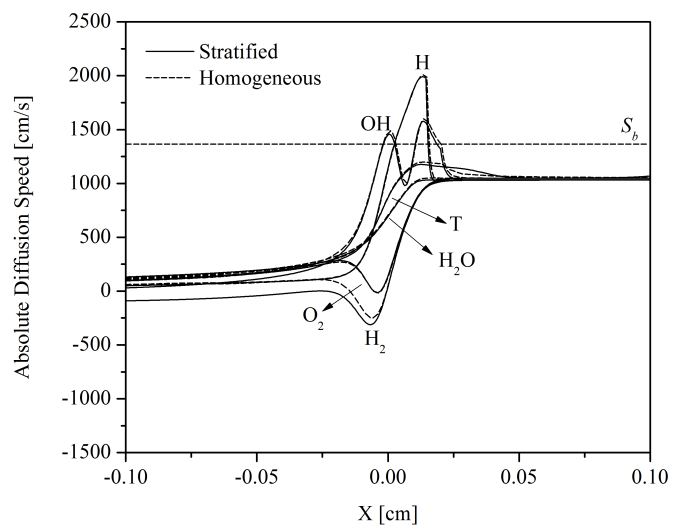

(c)

Figure 5: (a) heat release rate and temperature, (b) major species mole fraction and (c) thermal and species diffusion speeds profiles of SF and $\mathrm{HF}$ at $\phi_{f}=1.2$, when SF propagates from $\phi_{f}=2.0$ to $\phi_{f}=0.5, \delta_{S}=$ $0.1 \mathrm{~cm}$, with equal diffusivity assumption. 
Figure 6 compares the flame front propagation speeds and fluid expansion speeds of both SF and HF, and shows their respective differences $\left(\left.\Delta S\right|_{S F-H F}=\left.S\right|_{S F}-\right.$ $\left.S\right|_{H F}$ ). For both flame front propagation speeds and fluid expansion speeds, the same trends are observed: the results of SF are firstly lower than those of HF and then higher, similar to $0 \% S_{c}$ results. However, the differences in $S_{\text {exp }}$ between SF and HF, $\left.\Delta S_{\text {exp }}\right|_{S F-H F}$, are smaller than the differences in $S_{b}$ between SF and HF, $\left.\Delta S_{b}\right|_{S F-H F}$. Since local fuel consumption of SF and $\mathrm{HF}$ are identical, there must be additional effects besides burnt gas expansion that are responsible for the relatively large difference between $S_{b}$.

Figure 7 presents the velocity profiles of SF at several different time steps, where $\mathrm{x}$-axis stands for the spatial location of the 1D domain and y-axis is the corresponding fluid velocity. The sharp change of each curve is indicative of the flame front location. When the flame is located outside the stratification $\left(\phi_{f}=0.5\right.$ or 2.0$)$, burnt gas reaches steady state as the fluid velocity from the closed end to the flame is almost zero. However when SF propagates through the stratification, the burnt gas velocities deviate from zero. For example, at $\phi_{f}=1.7$ when the flame propagates from $\phi_{f}=2.0$ to 1.3 , while the unburnt gas in front of the flame front holds relatively slower speed, the flame front itself accelerates. As a result, not only is the unburnt gas further pushed away by the flame front, the burnt gas is also compressed towards the closed end, resulting in slightly negative fluid velocities as shown by the fluid velocity profile at $\phi_{f}=1.7$ in Figure 7. The flame front propagation speed is consequently smaller compared to that if the burnt gas is still. In comparison, when the flame propagates in the later part of stratification from $\phi_{f} 1.3$ to 0.5 , unburnt gas in front of the flame front still holds a higher speed while flame front itself has already slowed down. As a result, a contraction effect is created around: Instead of remaining still near the closed end, burnt gases are attracted towards the flame. As a result, burnt gases now have positive fluid velocities, as shown by in $\phi_{f}=0.9$ results in Figure 7. The fluid velocity at flame front is then increased, leading to an elevated flame front propagation speed. This hydrodynamic effect due to fluid continuity is caused by the variation of flame front propagation speeds across stratified charges with different equivalence ratios, which is only observed in stratified flames but not homogeneous flames. In short, this additional hydrodynamic effect also contributes to the difference in $S_{b}$ between SF and HF.

For lean-to-rich stratification case, where SF propagates from $\phi_{f}=0.5$ to $\phi_{f}=2.0$, the comparison of fuel consumption speeds and flame front propagation speeds are shown in Figure 8 and Figure 9 respectively. In Figure 8, while 5\% cutoff results are still identical, $0 \%$ result of SF is firstly lower and then higher than that of HF, as the burnt gas temperature of SF is still lower and then higher compared to that of HF. In Figure 9, both $S_{\text {exp }}$ and $S_{b}$ of SF are lower and then higher than those of HF, while the additional hydrodynamic effect due to fluid continuity is still present as $\left.\Delta S_{\text {exp }}\right|_{S F-H F}$ does not match $\left.\Delta S_{b}\right|_{S F-H F}$. Therefore, the same rational and analysis are still valid in this opposite stratification case.

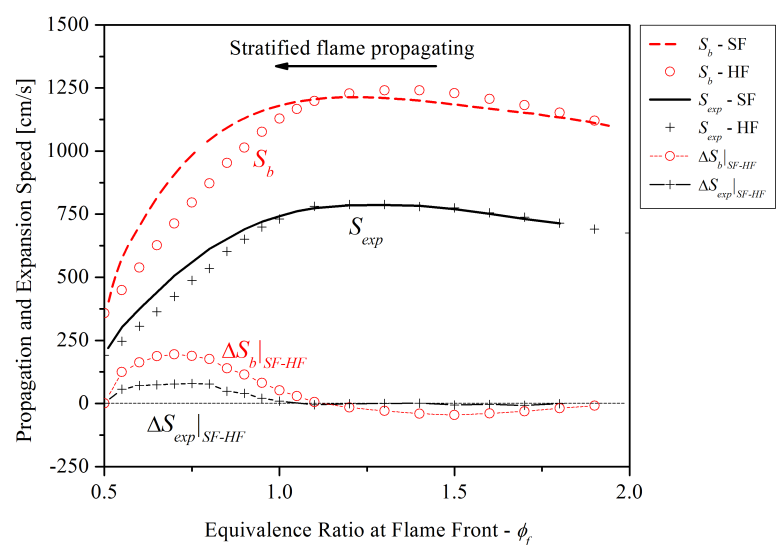

Figure 6: Flame front propagation speeds, fluid expansion speeds and respective differences between SF and HF, when SF propagates from $\phi_{f}=2.0$ to $\phi_{f}=0.5, \delta_{S}=0.1 \mathrm{~cm}$, with equal diffusivity assumption.

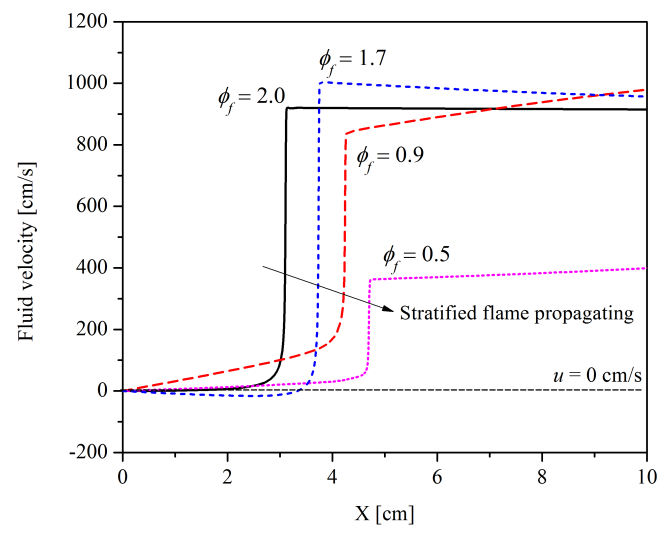

Figure 7: Fluid velocity profiles when SF propagates from $\phi_{f}=2.0$ to $\phi_{f}=0.5, \delta_{S}=0.1 \mathrm{~cm}$, with equal diffusivity assumption. 


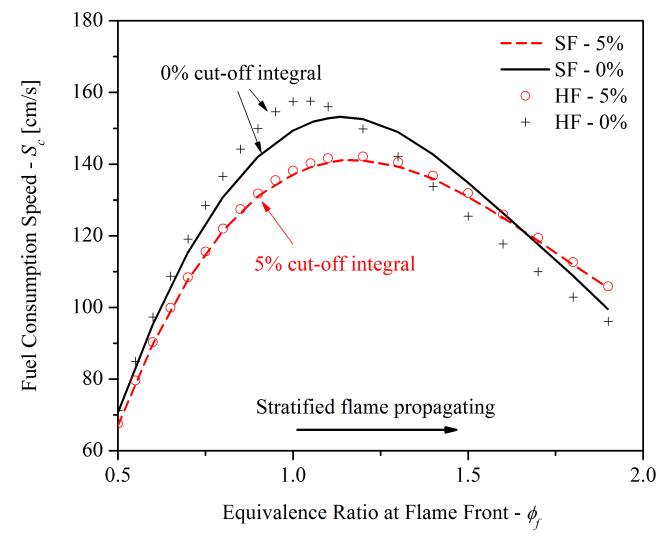

Figure 8: Fuel consumption speeds with $0 \%$ cutoff and $5 \%$ cutoff of $\mathrm{SF}$ and HF, when SF propagates from $\phi_{f}=0.5$ to $\phi_{f}=2.0, \delta_{S}=0.1$ $\mathrm{cm}$, with equal diffusivity assumption.

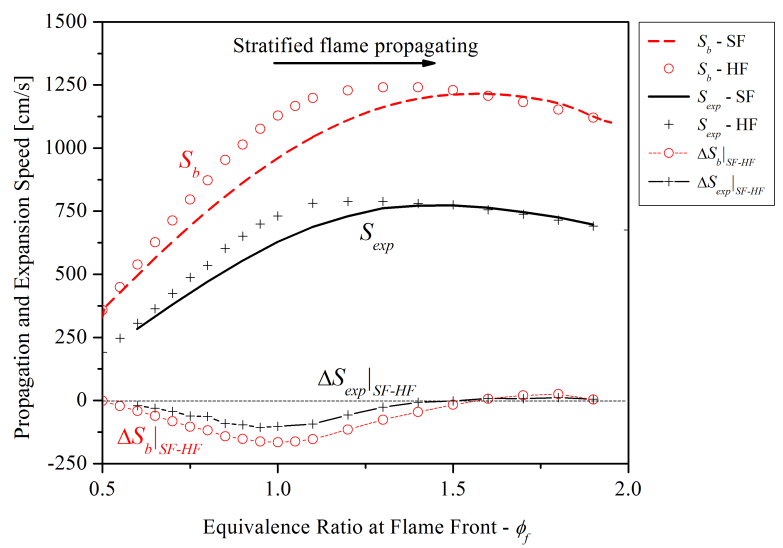

Figure 9: Flame front propagation speeds and fluid expansion speeds of SF and HF, when SF propagates from $\phi_{f}=0.5$ to $\phi_{f}=2.0, \delta_{S}=$ $0.1 \mathrm{~cm}$, with equal diffusivity assumption.

\subsection{Mixture-average Diffusivity Results}

Results with mixture-average diffusivity assumption are presented in this section. Each species has its own mass diffusivity which depends on temperature and the thermal differential diffusion (the Soret effect). Therefore, the Lewis number is not fixed: each species has its own Lewis number which changes at different positions in the flame. Due to preferential diffusion, lighter species, such as $\mathrm{H}, \mathrm{H}_{2}$, diffuse faster compared to heavier species. A similar set of numerical simulations as that with equal diffusivity assumption are investigated: the stratified flame propagates from unburnt charge of equivalence ratio 2.0 to that of 0.5 through the step change, as well as the opposite stratification case. Note that $\phi_{u}$ is no longer equal to $\phi_{f}$ due to preferential diffusion. In terms of $\phi_{f}$, the stratified flame is actually propagating from $\phi_{f}=1.6$ to 0.4 .

Figure 10 compares fuel consumption speed results calculated with both $0 \%$ and $5 \%$ cutoffs of SF and HF. Unlike the equal diffusivity results, both $0 \%$ cutoff and $5 \%$ cutoff results differ between SF and HF. More specifically, $5 \% S_{c}$ of SF is overall faster than that of $\mathrm{HF}$ across the entire stratification. Detailed flame characteristics of SF and $\mathrm{HF}$ at $\phi_{f}=1.0$ are investigated as shown in Figure 11. In Figure 11a, heat release rates near flame front of SF are higher than those of HF, indicating that local fuel consumption of SF is larger than that of HF. The temperature and species mole fraction profiles in Figure 11a and 11b show similar results as those in the case with equal diffusivity assumption: burnt gas temperature of SF is lower than that of HF. More $\mathrm{H}$ and $\mathrm{H}_{2}$ and less $\mathrm{OH}$ are found in the burnt gas of SF than HF, while they are almost identical near flame front. In Figure 11c, diffusion speed profiles are presented and significant differences arise compared to the corresponding case with equal diffusivity assumption: while thermal diffusion speed is still much lower than $S_{b}$, diffusion of $\mathrm{H}$ is able to catch up with the flame front propagation over a wide range across the flame front. Moreover, within the same range, $\mathrm{H}$ mole fraction in SF is notably higher than that of HF. Therefore compared to equal diffusivity case, $\mathrm{H}$ radical in burnt gas of SF will influence local fuel consumption speeds, referred as chemical effect. In this rich-to-lean stratified flame, more $\mathrm{H}$ radicals are generated in burnt gas of SF compared to HF. As a result, more $\mathrm{H}$ radicals will diffuse into the stratified flame and thus enhance the local chain-branching reactions, leading to enhancement of local flame speed near flame front. This local chemical effect agrees with the $5 \% S_{c}$ results as SF is always faster than that HF. Therefore, chemical effect is observed and has its impact on local flame characteristics with mixture-average diffusivity assumption, while thermal effect still does not exist. This observation is different from the conclusion drawn on methane/air stratified flames in [19]. As burnt gas fuel consumption is still dominated by burnt gas temperature, $0 \%$ cutoff results still follow the similar trend as seen in the corresponding equal diffusivity case.

Figure 12 compares the flame front propagation speeds and fluid expansion speeds of SF and HF, and shows their differences. Compared to equal diffusivity case, the difference in $S_{\text {exp }}$ between SF and HF, $\left.\Delta S_{\text {exp }}\right|_{S F-H F}$, is almost zero. Recall that with equal diffusivity assumption, fluid expansion effect results from heat release in the burnt gas and the difference between 


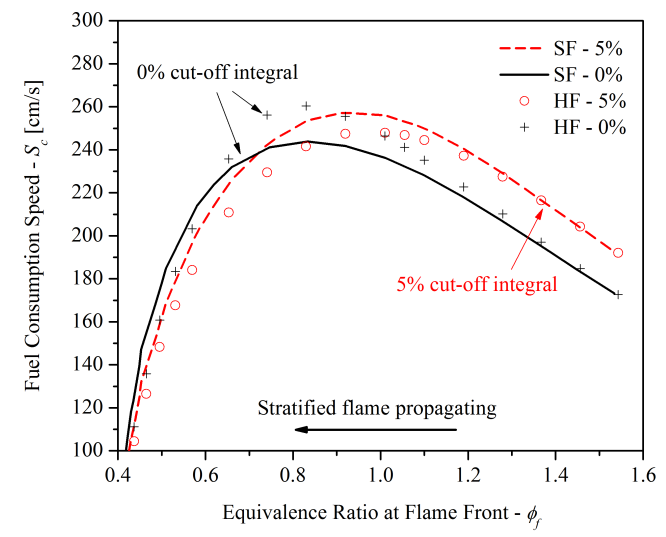

Figure 10: Fuel consumption speeds with $0 \%$ cutoff and $5 \%$ cutoff of $\mathrm{SF}$ and HF, when SF propagates from $\phi_{f}=1.6$ to $\phi_{f}=0.4, \delta_{S}=0.1$ $\mathrm{cm}$, with mixture-average diffusivity assumption.

SF and HF is due to different burnt gas compositions. In comparison with mixture-average diffusivity assumption, additional $\mathrm{H}$ radicals in stratified flames are more likely to diffuse towards the region of flame front due to preferential diffusion. As a result, the difference in burnt gas heat release between SF and HF as well as its contribution to flame front propagation speeds on flame front propagation speed becomes insignificant. As the flame is still burning at different speeds with different equivalence ratios, the hydrodynamic effect of fluid continuity is still present in SF. Therefore, the difference in $S_{b}$ is resulted from the chemical effect on local flame speeds and the hydrodynamic effect due to fluid continuity. In Figure 12, during the initial rich region, $S_{b}$ of $\mathrm{SF}$ is found slightly slower than HF due to the hydrodynamic effect. Afterwards $S_{b}$ of SF is overall faster than that of HF across the rest of the stratification due to faster local flame speeds.

The flame speed results of the opposite stratification case, where SF propagates from $\phi_{f}=0.4$ to $\phi_{f}=1.6$, are also investigated: As seen in Figure 13, 5\% $S_{c}$ of $\mathrm{SF}$ is overall lower than that of $\mathrm{HF}$, as less radicals diffuse into the local flame front of SF. $0 \% S_{c}$ of SF shows the trend of lower to higher than HF due to burnt gas temperature. In Figure 14, while $\left.\Delta S_{\text {exp }}\right|_{S F-H F}$ is still negligible, $S_{b}$ of SF is overall slower than that of $\mathrm{HF}$ across most of the stratification due to slower local flame speeds of SF, and slightly faster in the rich region due to hydrodynamic effect. Therefore, results from both stratification cases can be understood with the analysis of $5 \% S_{c}, 0 \% S_{c}$, species and fluid velocity profiles.

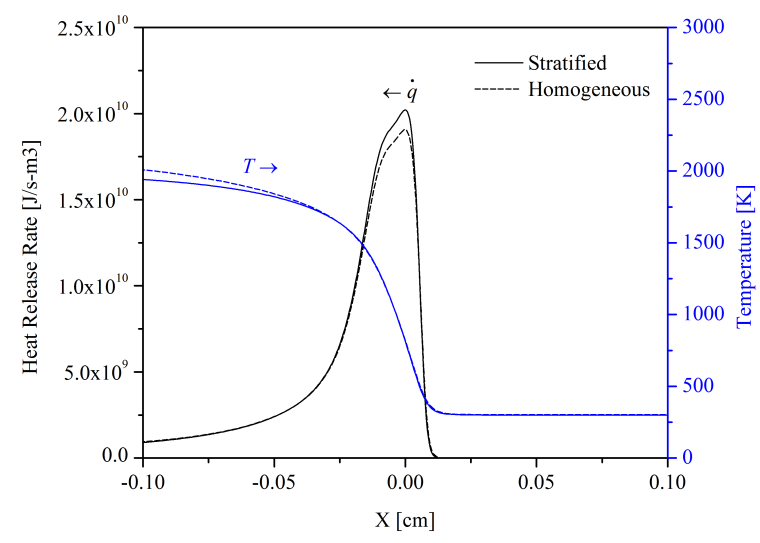

(a)

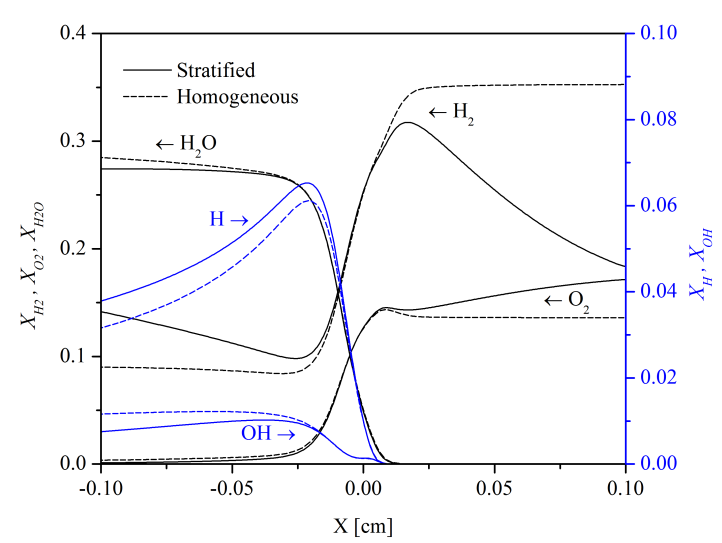

(b)

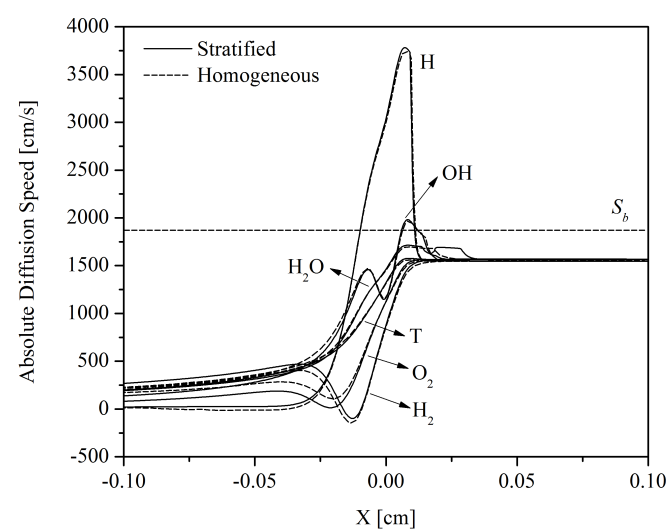

(c)

Figure 11: (a) heat release rate and temperature, (b) major species mole fraction and (c) thermal and species diffusion speeds profiles of SF and $\mathrm{HF}$ at $\phi_{f}=1.0$, when SF propagates from $\phi_{f}=1.6$ to $\phi_{f}=0.4, \delta_{S}=0.1 \mathrm{~cm}$, with mixture-average diffusivity assumption. 


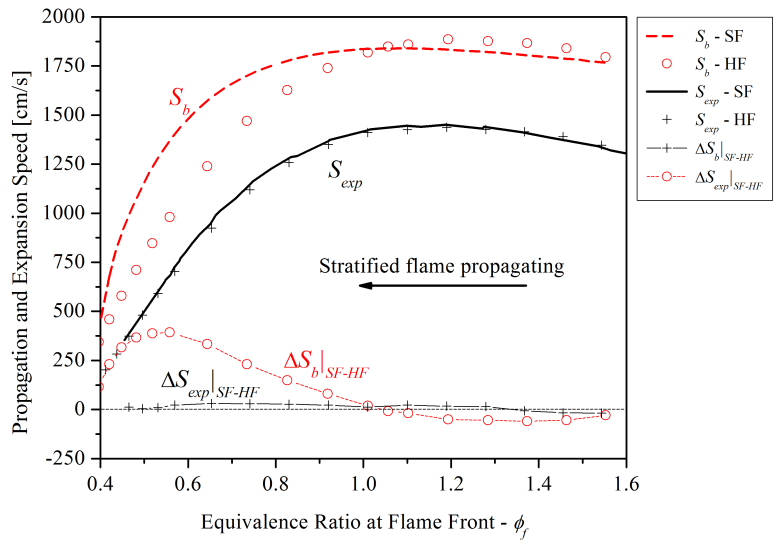

Figure 12: Flame front propagation speeds and fluid expansion speeds of SF and HF, when SF propagates from $\phi_{f}=1.6$ to $\phi_{f}=0.4, \delta_{S}=$ $0.1 \mathrm{~cm}$, with mixture-average diffusivity assumption.

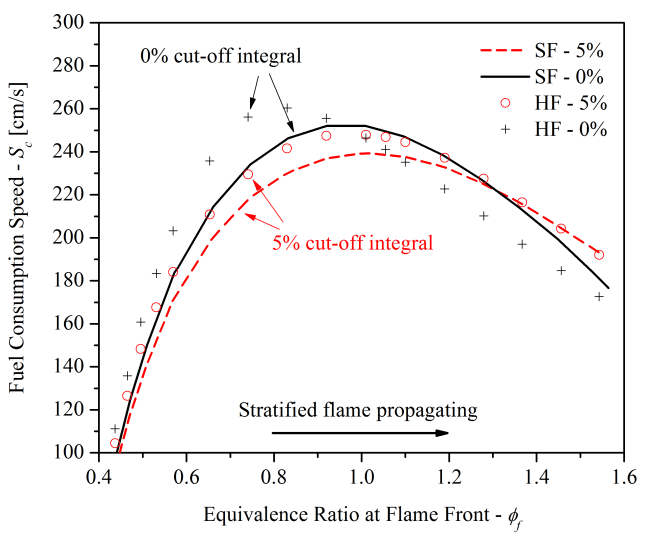

Figure 13: Fuel consumption speeds with $0 \%$ cutoff and $5 \%$ cutoff of $\mathrm{SF}$ and HF, when SF propagates from $\phi_{f}=0.4$ to $\phi_{f}=1.6, \delta_{S}=0.1$ $\mathrm{cm}$, with mixture-average diffusivity assumption.

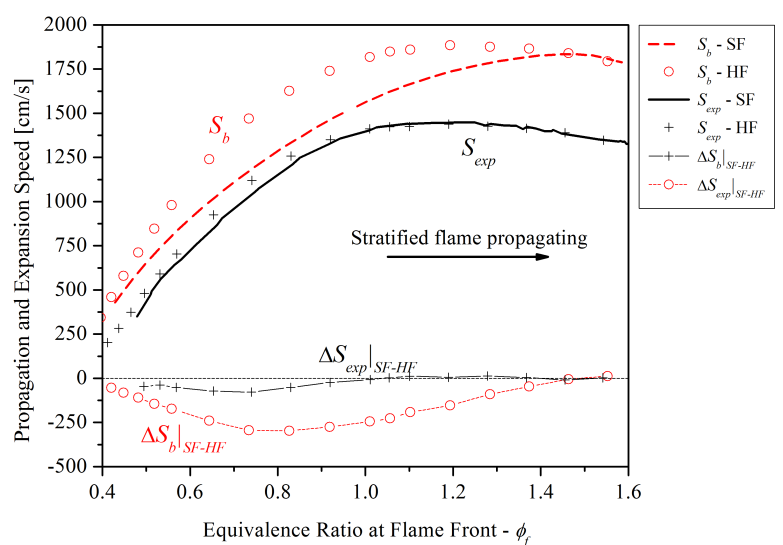

Figure 14: Flame front propagation speeds and fluid expansion speeds of SF and HF, when SF propagates from $\phi_{f}=0.4$ to $\phi_{f}=1.6, \delta_{S}=$ $0.1 \mathrm{~cm}$, with mixture-average diffusivity assumption.

\subsection{Effect of Stratification Thickness}

Stratified flames with different stratification thicknesses, ranging from the order of flame thickness to relatively large distance (approximately 20 times larger than the flame thickness), are compared to homogeneous flames, with both equal and mixture-average diffusivity assumptions. Figures 15 and 16 show the effect of stratification thickness on $5 \% S_{c}$ and $S_{b}$ for the stratified flame propagating from $\phi_{f}=2.0$ to $\phi_{f}=0.5$ with equal diffussivity assumption. While no difference is observed between $5 \% S_{c}$ of SF and HF when $\delta_{S}=0.1$ $\mathrm{cm}$ and $\delta_{S}=1 \mathrm{~cm}$, SF with $\delta_{S}=0.04 \mathrm{~cm}$ is overall slightly faster than $\mathrm{HF}$, similar to previous mixtureaverage diffusivity results. Similar to previous analysis, Figure 17 shows the heat release rate, temperature, species mole fraction and their corresponding diffusion speeds profiles of SF with $\delta_{S}=0.04 \mathrm{~cm}$ and $\mathrm{HF}$ when $\phi_{f}=1.2$. As the difference between $5 \% S_{c}$ is quite small, the difference between heat release rates in Figure $17 \mathrm{a}$ is similarly insignificant. Although the temperature profiles especially in the burnt gas are notebly different, heat diffusion speed is still not fast enough to catch up with the flame as shown in Figure 17c, indicating no thermal effect. In the meanwhile, the difference between $\mathrm{H}$ radical profiles in Figure $17 \mathrm{~b}$ is enlarged and further expanded to the flame location compared to larger $\delta_{S}$ results in Figure 5b. As a result, extra $\mathrm{H}$ radicals in burnt gas of SF are able to diffuse into the flame and enhance the local flame speed. Therefore, when $\delta_{S}$ is reduced to the order of flame thickness, weak chemical effect is observed even with equal diffusivity assumption. $S_{b}$ of SF at different $\delta_{S}$ are always different from $\mathrm{HF}$ due to hydrodynamics effects. With increasing $\delta_{S}$, divergence between $S_{b}$ of SF and HF decreases. 


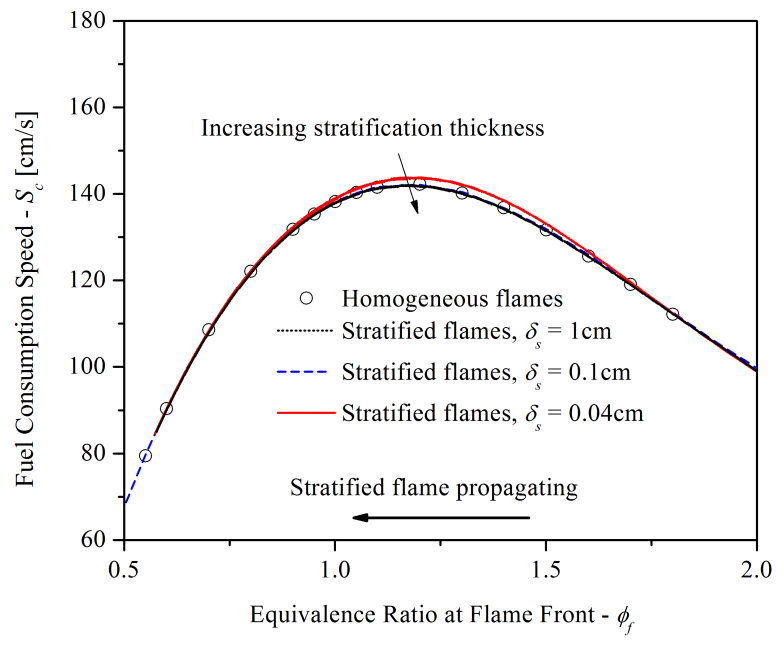

Figure 15: Fuel consumption speeds with 5\% cutoff of SF at different stratification thickness and corresponding HF, when SF propagates from $\phi_{f}=2.0$ to $\phi_{f}=0.5$, with equal diffusivity assumption.

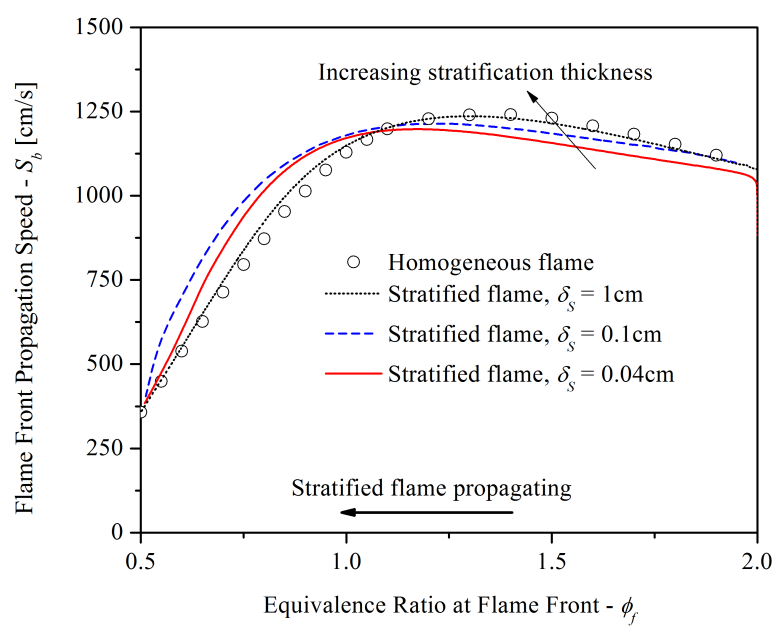

Figure 16: Flame front propagation speeds of SF at different stratification thickness and corresponding HF, when SF propagates from $\phi_{f}=2.0$ to $\phi_{f}=0.5$, with equal diffusivity assumption.

Figures 18 and 19 show the influence of stratification thickness on $5 \% S_{c}$ and $S_{b}$ for the stratified flame propagating from $\phi_{f}=1.6$ to $\phi_{f}=0.4$ with mixtureaverage diffussivity assumption. Both $S_{c}$ and $S_{b}$ of SF with different $\delta_{S}$ are different from those of HF. When $\delta_{S}$ is $0.04 \mathrm{~cm}$, both $S_{c}$ and $S_{b}$ results show considerably large differences between SF and HF. Therefore, preferential diffusion of $\mathrm{H}$ radical further enhances chemical effect, as it is observed over a much wider range of stratification thicknesses and at larger magnitudes. As $\delta_{S}$ increases, both $S_{c}$ and $S_{b}$ of SF approach their corresponding results of HF.

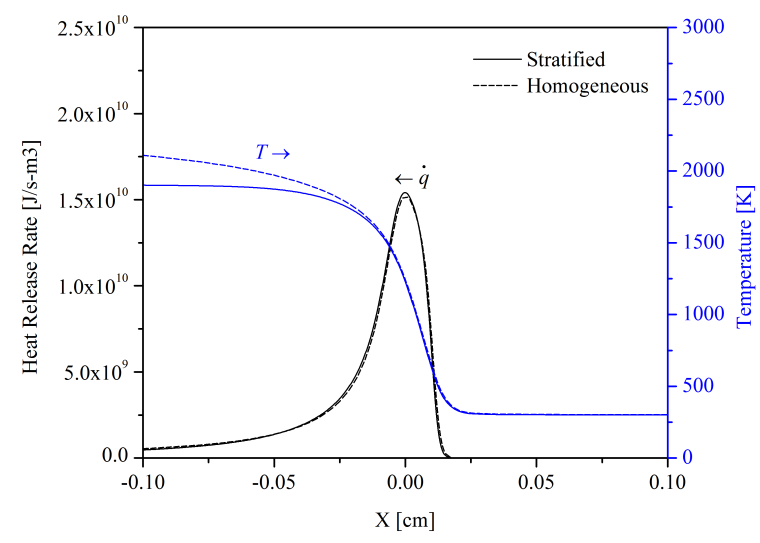

(a)

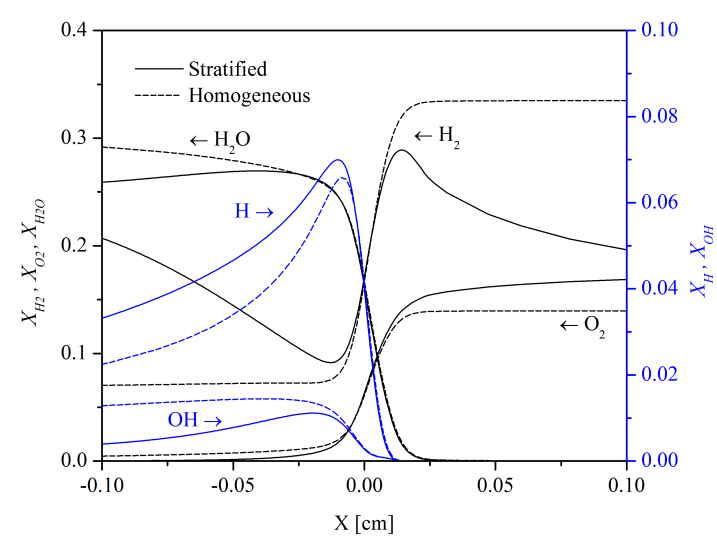

(b)

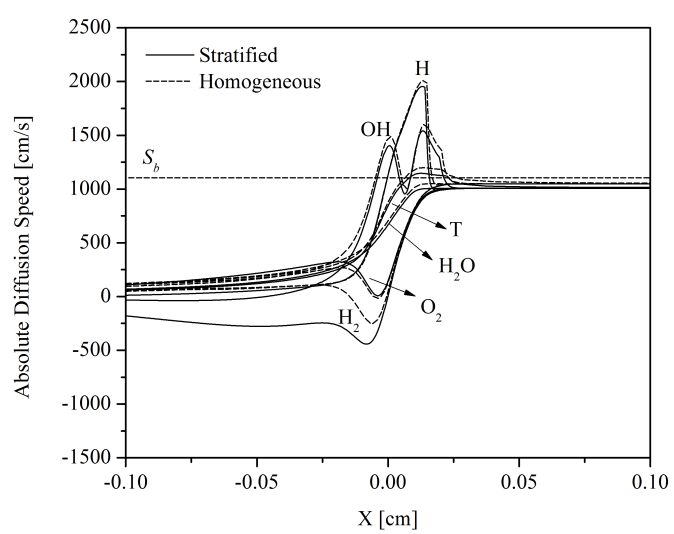

(c)

Figure 17: (a) heat release rate and temperature, (b) major species mole fraction and (c) thermal and species diffusion speeds profiles of SF and $\mathrm{HF}$ at $\phi_{f}=1.2$, when SF propagates from $\phi_{f}=2.0$ to $\phi_{f}=0.5, \delta_{S}=0.04 \mathrm{~cm}$, with equal diffusivity assumption. 


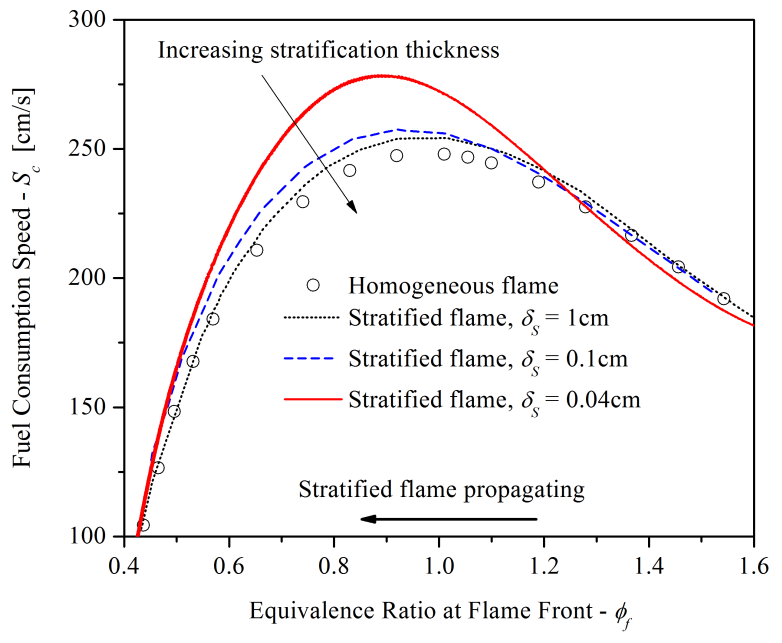

Figure 18: Fuel consumption speeds with 5\% cutoff of SF at different stratification thickness and corresponding HF, when SF propagates from $\phi_{f}=1.6$ to $\phi_{f}=0.4$, with mixture-average diffussivity assumption.

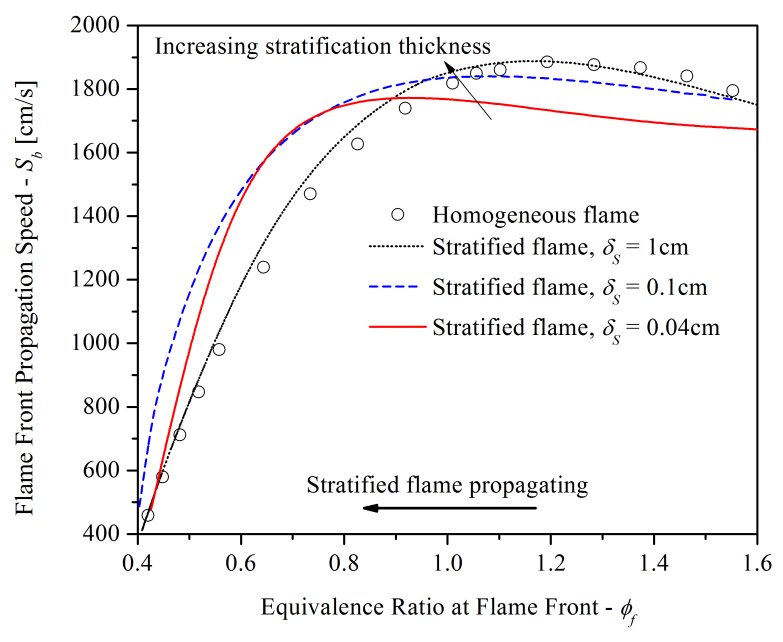

Figure 19: Flame front propagation speeds of SF at different stratification thickness and corresponding $\mathrm{HF}$, when SF propagates from $\phi_{f}=1.6$ to $\phi_{f}=0.4$, with mixture-average diffussivity assumption.

For local fuel consumption speeds, larger stratification thickness reduces the difference between burnt gas composition of SF and HF. As a result, less difference in burnt gas heat release between SF and HF is achieved. Local chemical effect is therefore suppressed leading to smaller difference in $S_{c}$ between SF and HF. For flame front propagation speeds, larger stratification thickness reduces the magnitude of fluid velocity variation so that the hydrodynamic effect due to continuity is also decreased. As both local chemical and hydrodynamic effects are weakend, difference in $S_{b}$ between $\mathrm{SF}$ and $\mathrm{HF}$ becomes smaller. In conclusion, with increasing stratification thickness, both local chemical and hydrodynamic effects decrease.

\subsection{Lean Flammability Extension}

According to literature, the lean flammability limit of homogeneous hydrogen/air flame under ambient temperature and atmospheric pressure is measured at equivalence ratio $\phi_{u}=0.4[38,39]$. Therefore, the flame characteristics of HF at $\phi_{u}=0.4$ is used as the threshold to decide the flammability limit in this numerical study. Note that due to preferential diffusion, $\phi_{u}=0.4$ is equivalent to $\phi_{f}=0.356$.

One stratified flame case with mixture-average diffussivity assumption, analogous to the practical situation in direct injection engines, is performed. The stratified flame is propagating from unburnt mixture at $\phi_{u}=1.0$ to $\phi_{u}=0.2$ with $\delta_{S}=0.1 \mathrm{~cm}$. Figure 20a compares the major species and radical profiles of both SF and HF at lean flammability $\phi_{f}=0.356$. Even though the overall concentrations of species and radicals are low as $\phi_{f}$ is at flammability limit, SF still has relatively larger amount of $\mathrm{H}$ and $\mathrm{OH}$ radicals at flame front as well as in the burnt gas, which can enhance the local flame burning. This conclusion can be supported by Figure 20b, showing the total heat release rate and major exothermic reaction rates of SF and $\mathrm{HF}$ at $\phi_{f}=0.356$. SF has overall more heat release than $\mathrm{HF}$ and major exothermic reactions are indeed related to $\mathrm{H}$ and $\mathrm{OH}$ radicals; therefore $\mathrm{SF}$ has not reached the lean flammability yet.

As SF continues to burn leaner, Figure 21a and 21b show the results of SF at $\phi_{f}=0.346$ and $\mathrm{HF}$ at $\phi_{f}=$ 0.356 . The radical and reactant profiles near flame front as well as heat release profiles match exactly with each other between SF and HF. Therefore it is reasonable to conclude that SF has reached its lean flammability limit at $\phi_{f}=0.346$. The extension of lean flammability limit of SF, from $\phi_{f}=0.356$ to $\phi_{f}=0.346$ is achieved by local chemical effect, but relatively insignificant. As the overall radical and reactant concentrations are quite low near flammability, the overall reactivity is as well low so that the local chemical effect is significantly weakened. Furthermore, since the flame is propagating really slow near the lean flammability, it gives significantly more time for the stratification layer to diffuse. When the stratified flame reaches the lean flammability limit, $\delta_{S}$ has grown to the order of $1 \mathrm{~cm}$ from its original $0.1 \mathrm{~cm}$. 


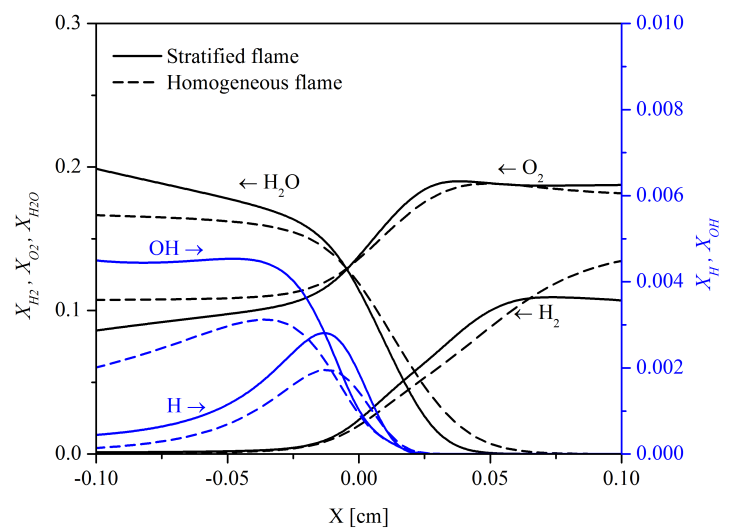

(a)

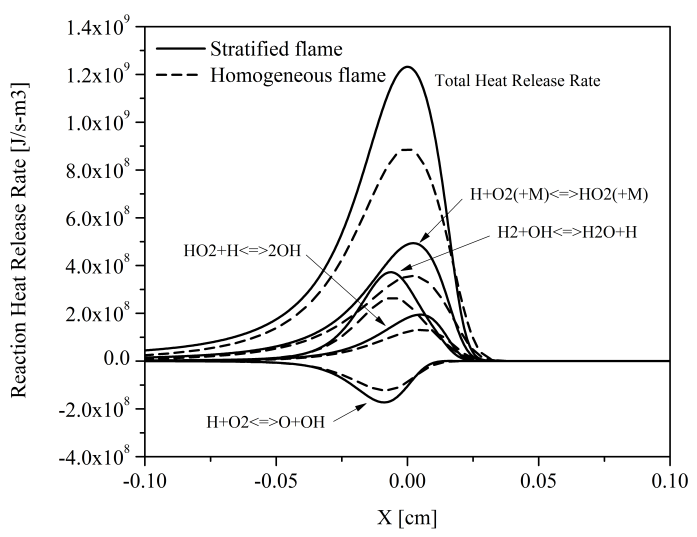

(b)

Figure 20: (a)major species mole fraction and (b)heat release rate and major exothermic reaction rate profiles of SF and $\mathrm{HF}$ at $\phi_{f}=0.356$, when SF propagates from $\phi_{u}=1.0$ to $\phi_{u}=0.4, \delta_{S}=0.1 \mathrm{~cm}$, with mixture-average diffusivity assumption.

\section{Conclusions}

Detailed numerical studies have been performed on hydrogen/air stratified flames in 1-D planar coordinate using A-SURF. Stratified flames propagate through a natural equivalence ratio gradient where burnt gas temperature and composition are different from those of corresponding HF, while investigated stratification thicknesses are at the same order of magnitude or larger than flame thickness. In addition to comparison of $0 \%$ fuel consumption speeds and flame front propagation speeds, new insights of how stratification influences laminar flame speed are gained by analyzing local fuel consumption, burnt gas expansion, diffusion speeds of heat and species and hydrodynamic effect due to fluid

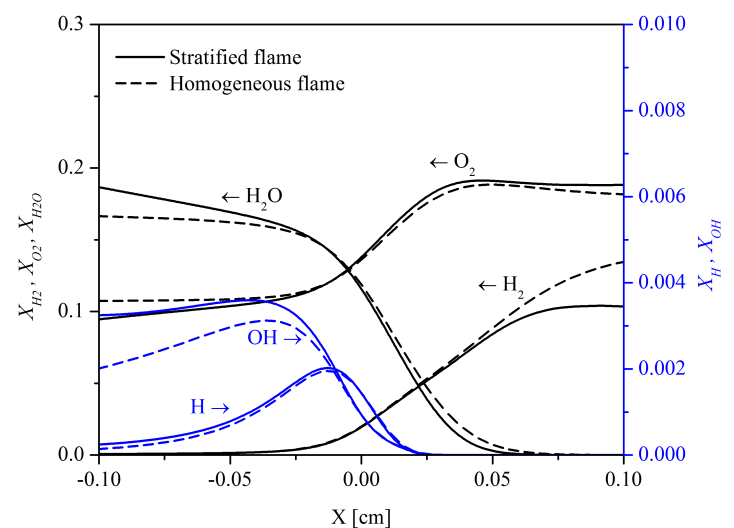

(a)

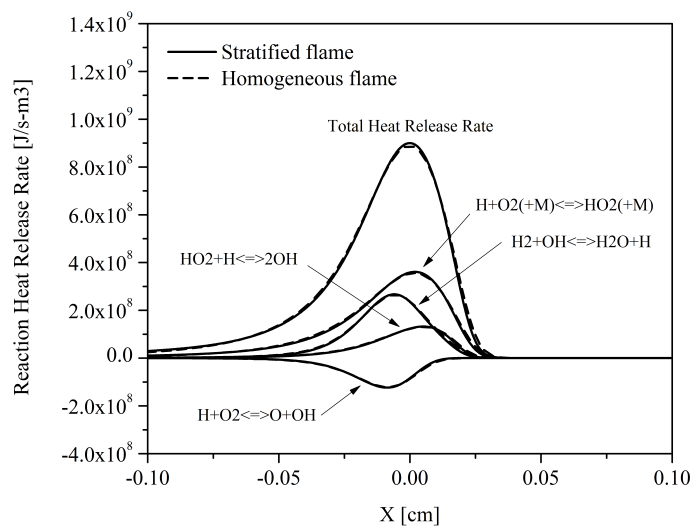

(b)

Figure 21: (a)major species mole fraction and (b)heat release rate and major exothermic reaction rate profiles of $\mathrm{HF}$ at $\phi_{\text {local }}=0.356$ and $\mathrm{SF}$ at $\phi_{\text {local }}=0.346$, when SF propagates from $\phi_{u} 1.0$ to $0.2, \delta_{S}=0.1 \mathrm{~cm}$, with mixture-average diffusivity assumption.

velocity variation. The following specific conclusions have been reached from the observations and analysis:

- With equal diffusivity assumption, as 5\% cutoff fuel consumption speeds are identical between SF and HF when stratification thickness is larger than flame thickness, neither thermal effect nor chemical effect is seen locally near flame front. When stratification thickness reduces to the order of flame thickness, weak chemical effect due to different level of $\mathrm{H}$ radical in burnt gases is observed even with equal diffusivity assumption. As burnt gas continues to react after the flame has passed through, the difference between $0 \%$ cutoff fuel consumption speeds are resulted from burnt 
gas fuel consumption dominated by burnt gas temperature. The difference between flame front propagation speeds are caused by fluid expansion due to burnt gas heat release as well as the hydrodynamic effect due to fluid continuity.

- With mixture-average diffusivity assumption, chemical effect due to different level of $\mathrm{H}$ radicals in burnt gas is observed in stratified flames as $5 \%$ cutoff fuel consumption speed of rich-to-lean $\mathrm{SF}$ is overall faster than the corresponding HF. The chemcial effect is further enhanced by preferential diffusion of $\mathrm{H}$ radical. Lean-to-rich $\mathrm{SF}$ is respectively slower than HF. 0\% cutoff fuel consumption speeds are still influenced by burnt gas fuel consumption. As additional $\mathrm{H}$ radicals generated from burnt gas fuel consumption in SF diffuse from the burnt gas into the flame region, the difference in burnt gas heat release between $\mathrm{SF}$ and HF becomes insignificant. The difference between flame front propagation speeds are thereby resulted from the difference between local flame speeds due to chemical effect in addition to the hydrodynamic effect due to fluid continuity.

- Two transport models respond differently to different stratification thicknesses: with equal diffusivity assumption, weak chemical effect is observed only when stratification thickness is reduced to the order of flame thickness. In comparison, with mixture average diffusivity assumption, chemical effect is observed in a wider range of stratification thicknesses up to $1 \mathrm{~cm}$, as preferential diffusion of $\mathrm{H}$ radical further enhances the chemical effect. Overall, increasing stratification thickness leads to decreased gradients in burnt gas compositions as well as decrease in the changing rate of flame front propagation speeds. Thus both local chemical and hydrodynamic effects are reduced.

- No significant lean flammability extension is observed for hydrogen/air stratified flames, as chemical effect is significantly weakened due to overall low reactivity and enlarged stratification thickness.

This study provides physical mechanism and numerical data references for development of predictive models for laminar flame speeds of stratified flames. Using the same methodology, different fuel/air mixtures can be investigated. Different results are expected due to differences in transport, chemistry and mass diffusivity of reactants and radicals.

\section{Acknowledgments}

This work at the University of California, Berkeley was supported by the National Science Foundation and U.S. Department of Energy under award CBET1258653. This work at Peking University was supported by the National Natural Science Foundation of China No.51322602.

\section{References}

[1] R. D. Reitz, Directions in internal combustion engine research, Combustion and Flame 160 (1) (2013) 1-8.

[2] A. C. Alkidas, Combustion advancements in gasoline engines, Energy Conversion and Management 48 (2007) 2751-2761.

[3] C. Park, S. Kim, H. Kim, Y. Moriyoshi, Stratified lean combustion characteristics of a spray-guided combustion system in a gasoline direct injection engine, Energy 41 (1) (2012) 401-407.

[4] M. Aliramezani, I. Chitsaz, A. A. Mozafari, Thermodynamic modeling of partially stratified charge engine characteristics for hydrogen-methane blends at ultra-lean conditions, International Journal of Hydrogen Energy 38 (2013) 10640-10647.

[5] D. Dahl, M. Andersson, I. Denbratt, The role of charge stratification for reducing ringing in gasoline engine homogeneous charge compression ignition combustion investigated by optical imaging, International Journal of Engine Research 14 (2013) 525-536.

[6] M. Sjöberg, J. E. Dec, Smoothing HCCI Heat-Release Rates Using Partial Fuel Stratification with Two-Stage Ignition Fuels, SAE Technical Paper 2006-01-0629.

[7] Z. Zheng, M. Yao, Charge stratification to control HCCI: Experiments and CFD modeling with n-heptane as fuel, Fuel 88 (2) (2009) 354-365.

[8] S. Chung, Stabilization, propagation and instability of tribrachial triple flames, Proceedings of the Combustion Institute 31 (1) (2007) 877-892.

[9] H. G. Im, J. H. Chen, Structure and propagation of triple flames in partially premixed hydrogen-air mixtures, Combustion and Flame 119 (4) (1999) 436-454.

[10] H. Guo, F. Liu, G. J. Smallwood, A numerical study on NOx formation in laminar counterflow CH4/air triple flames, Combustion and Flame 143 (3) (2005) 282-298.

[11] D. G. Talley, Some properties of nearly premixed laminar flame propagation along weakly stratified layers in combustible gas mixtures, Combustion and Flame 79 (1990) 141-150.

[12] R. Sankaran, H. G. Im, Dynamic flammability limits of methane/air premixed flames with mixture composition fluctuations, Proceedings of the Combustion Institute 29 (2002) 77-84.

[13] P. Cisse, G. a. Karim, The rapid formation and dispersion of flammable zones within cylindrical vertical enclosures following the release of a fixed mass of hydrogen and other gaseous fuels into air, International Journal of Hydrogen Energy 32 (2007) 630-636.

[14] S. Balusamy, A. Cessou, B. Lecordier, Laminar propagation of lean premixed flames ignited in stratified mixture, Combustion and Flame 161 (2014) 427-437.

[15] O. Badr, G. Karim, Flame Propagation in Stratified Methane-Air Mixtures, Journal of Fire Sciences 2 (1984) 415-426.

[16] P. C. Vena, B. Deschamps, G. J. Smallwood, M. R. Johnson, Equivalence ratio gradient effects on flame front topology in a stratified iso-octane/air turbulent V-flame, Proceedings of the Combustion Institute 33 (2011) 1551-1558. 
[17] J. S. Kim, J. Park, O. B. Kwon, D. S. Bae, J. H. Yun, S. I. Keel, A study on flame structure and extinction in downstream interaction between lean premixed $\mathrm{CH} 4$-air and $(50 \% \mathrm{H} 2+50 \% \mathrm{CO})$ syngas-air flames, International Journal of Hydrogen Energy 36 (2011) 5717-5728.

[18] R. Owston, J. Abraham, Flame propagation in stratified hydrogen-air mixtures: Spark placement effects, International Journal of Hydrogen Energy 34 (2009) 6532-6544.

[19] A. D. Cruz, A.M.Dean, J.M.Grenda, A numerical study of the laminar flame speed of stratified methane/air flames, Proceedings of the Combustion Institute 28 (2000) 1925-1932.

[20] R. Zhou, S. Hochgreb, The behaviour of laminar stratified methane/air flames in counterflow, Combustion and Flame 160 (2013) 1070-1082.

[21] M. Kuznetsov, S. Kobelt, J. Grune, T. Jordan, Flammability limits and laminar flame speed of hydrogen-air mixtures at subatmospheric pressures, International Journal of Hydrogen Energy 37 (2012) 17580-17588.

[22] A. E. Dahoe, Laminar burning velocities of hydrogen air mixtures from closed vessel gas explosions, Journal of Loss Prevention in the Process Industries 18 (2005) 152-166.

[23] Z. Chen, Studies on the Initiation , Propagation , and Extinction of Premixed Flames, Ph.D. thesis (2009).

[24] M. P. Burke, M. Chaos, Y. Ju, F. L. Dryer, S. J. Klippenstein, Comprehensive H 2/O 2 kinetic model for high-pressure combustion, International Journal of Chemical Kinetics 44 (7) (2012) 444-474.

[25] R. J. Kee, F. M. Rupley, J. A. Miller, Chemkin-ii: A fortran chemical kinetics package for the analysis of gas-phase chemical kinetics, Tech. rep., Sandia National Labs., Livermore, CA (USA) (1989).

[26] Z. Chen, M. P. Burke, Y. Ju, Effects of Lewis number and ignition energy on the determination of laminar flame speed using propagating spherical flames, Proceedings of the Combustion Institute 32 I (2009) 1253-1260.

[27] Z. Chen, Effects of radiation and compression on propagating spherical flames of methane/air mixtures near the lean flammability limit, Combustion and Flame 157 (2010) 2267-2276.

[28] P. Dai, Z. Chen, S. Chen, Y. Ju, Numerical experiments on reaction front propagation in n-heptane/air mixture with temperature gradient, Proceedings of the Combustion Institute 35 (2014) 3045-3052.

[29] E. Varea, J. Beeckmann, H. Pitsch, Z. Chen, B. Renou, Determination of burning velocities from spherically expanding H2/air flames, Proceedings of the Combustion Institute 35 (2015) 711719.

[30] R. J. Kee, J. F. Grcar, M. Smooke, J. Miller, E. Meeks, Premix: a fortran program for modeling steady laminar one-dimensional premixed flames, Sandia National Laboratories Report.

[31] E. Hu, Z. Huang, J. He, C. Jin, J. Zheng, Experimental and numerical study on laminar burning characteristics of premixed methane-hydrogen-air flames, International Journal of Hydrogen Energy 34 (2009) 4876-4888.

[32] C. M. Vagelopoulos, J. H. Frank, J. Jeffries, F. Dinkelacker, An experimental and numerical study on the adequacy of $\mathrm{CH}$ as a flame marker in premixed methane flames, Proceedings of the Combustion Institute 30 I (2005) 241-249.

[33] C. Law, C. Sung, Structure, aerodynamics, and geometry of premixed flamelets, Progress in Energy and Combustion Science 26 (4) (2000) 459-505.

[34] C. K. Law, Combustion physics, Cambridge University Press, 2006.

[35] T. Poinsot, T. Echekki, M. G. Mungal, A Study of the Laminar Flame Tip and Implications for Premixed Turbulent Combustion, Combustion Science and Technology 81 (1992) 45-73.
[36] T. Poinsot, D. Veynante, Theoretical and numerical combustion, RT Edwards, Inc., 2005.

[37] F. Wu, W. Liang, Z. Chen, Y. Ju, C. K. Law, Uncertainty in stretch extrapolation of laminar flame speed from expanding spherical flames, Proceedings of the Combustion Institute 35 (1) (2014) 663-670.

[38] W. Braker, A. L. Mossman, Matheson gas data book, Matheson East Rutherford, NJ, 1980.

[39] M. G. Zabetakis, Flammability characteristics of combustible gases and vapors, Tech. rep., DTIC Document (1965). 